\title{
Ultra Low-Dispersion Spectroscopy with Gaia and Photographic Objective Prism Surveys
}

\author{
R. Hudec, L. Hudec, M. Klíma
}

\begin{abstract}
This paper discusses the ultra low-dispersion spectroscopy to be applied in the ESA Gaia space observatory and the ground-based objective-prism plate surveys. Although the dispersion in plate surveys is usually larger than in the Gaia $\mathrm{BP} / \mathrm{RP}$ spectrometers, the spectral resolutions differ by a factor of $2-3$ only, since the resolution in ground-based spectra is seeing-limited. We argue that some of the algorithms developed for digitized objective-prism plates can also be applied for the Gaia spectra. At the same time, the plate results confirm the feasibility of observing strong emission lines with Gaia RP/BP.
\end{abstract}

Keywords: astronomical plates, astronomy satellites, ESA Gaia, low-dispersion spectroscopy.

\section{Introduction}

The ESA Gaia satellite payload consists of a single integrated instrument, the design of which is characterised by a dual telescope concept with a common structure and a common focal plane (http://sci.esa.int/gaia/). Both telescopes are based on a three-mirror anastigmat (TMA) design. Beam combination is achieved in image space with a small beam combiner. Silicon-carbide (SiC) ultra-stable material is used for the mirrors and the telescope structure. There will be a large common focal plane with an array of 106 CCDs. The large focal plane also includes areas dedicated to the spacecraft's metrology and alignment measurements. Three instrument functions/modes are designed: (i) Astrometric mode for accurate measurements, even in densely populated sky regions of up to 3 million $\operatorname{stars} / \mathrm{deg}^{2}$, (ii) Photometric mode based on low-resolution, dispersive spectro-photometry using Blue and Red Photometers (BP and RP) for continuous spectra in the 320-1000 nm band for astrophysics and chromaticity calibration of the astrometry, and (iii) Spectrometroscopic (RVS) mode for high resolution, with grating, covering a narrow band: $847-874 \mathrm{~nm}$. The expected limiting magnitude is 20 in photometric mode (http://sci.esa.int/gaia). See also Figure 1.

In our study we focus on the "photometric mode" $\mathrm{RP} / \mathrm{BP}$. Use of the dispersive element (prism) generates ultra low-dispersion spectra. One disperser, called BP for Blue Photometer, operates in the 330 $660 \mathrm{~nm}$ wavelength range; the other, called RP for Red Photometer, covers the 650-1000 nm wavelength range. The dispersion is higher at short wavelengths, and ranges from 4 to $32 \mathrm{~nm} /$ pixel for $\mathrm{BP}$ and from 7 to $15 \mathrm{~nm} /$ pixel for RP (http://sci.esa.int/gaia). It should be noted however that the photometric CCDs are located at the edge of the focal plane, where the quality of the images is more sensitive to aberrations than astrometric images (Straizys, 2010).

The spectral coverage of Gaia, i.e. the $G$ band, and RP, BP and RVS passbands is illustrated in Figure 1 .

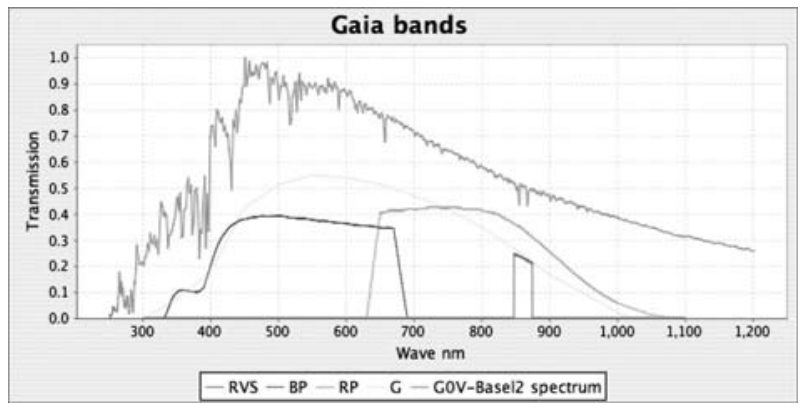

Fig. 1: The spectral coverage by Gaia (http://sci.esa.int/gaia)

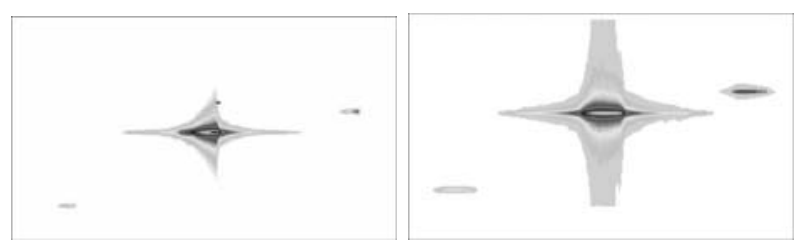

Fig. 2: BP (left) and BR (right) images simulated by the GIBIS simulator, the same sky field. The images have different scale parameter values (visualization DS9). These images illustrate the image wings mentioned by Straizys et al., 2006

The BP and RP spectra will be binned on-chip in the across-scan direction; no along-scan binning is foreseen. RP and BP will be able to reach ob- 
ject densities on the sky of at least 750,000 objects $\mathrm{deg}^{-2}$. The obtained complex images can be simulated by the GIBIS simulator (Figure 2). GIBIS is a pixel-level simulator of the Gaia mission intended to simulate how the Gaia instruments will observe the sky, using realistic simulations of the astronomical sources and of the instrumental properties. It is a branch of the global Gaia Simulator (GaiaSimu) under development within Gaia Coordination Unit 2: Data Simulations.

We note that certain types of variable stars (VS) such as Miras, Cepheids, and a few cases of other stars, mostly peculiar variables, exhibit large variations in their spectral types. This field is, however, little exploited, as these studies used to be very laborious (plates were mostly visually inspected) and limited, and hence no review on the spectral variability among VS exists. The ESA Gaia is expected to deliver data to fill this gap.

An important question is whether the dispersion of these devices is enough to detect and to study bright spectral features/emission lines. We also briefly introduce the extended work of US astrophysicist and NASA Astronaut Karl Henize, who spent a large part of his scientific career on low dispersion spectroscopy with objective prism. We have found the 290 large $15 \times 15$ inch original low-dispersion spectral plates that he took about 60 years ago in South Africa and analysed extensively. We found and investigated these plates (probably the complete Henize collection) in the PARI (Pisgah Astronomical Research Institute) Institute, NC, USA (Figure 3). The plates show numerous examples of objects with very prominent emission lines, which he found in a very extended time-consuming and laborious project (Figure 4).

\section{Ultra low dispersion spectral plate databases}

LDS (Low-Dispersion Spectroscopy) astrophysics was evolved and performed at numerous observatories between ca 1930 and 1980. Mostly LDS with
Schmidt telescopes (plates with objective prism) were used for various projects e.g. QSO, emission line and Halpha surveys, star classifications, etc. The technique was however little used after 1980.

Some of these surveys are listed below (the dispersion data is given in the next section, and we also note that many other similar surveys exist):

(1) Schmidt Sonneberg Camera. Sky survey (selected fields) with a $50 / 70 \mathrm{~cm}$ Schmidt telescope. No online access yet but the scans can be provided upon request (http://www.stw.tu-ilmenau.de/).

(2) Bolivia Expedition Spectral Plates. These plates offer homogeneous but not full coverage (90 southern Kapteyn's Selected Areas Nos. 116-206 were covered with plates representing $10 \times 10 \mathrm{grad}$ each, hence 9000 square degrees in total) of the southern sky with spectral and direct plates, directed by the Potsdam Observatory. The plates are stored at the Sonneberg Observatory (http://www.stw.tuilmenau.de/) and were taken between 1926-1928, in total about 70000 prism spectra were estimated and published in Potsdam Publications, see Becker, 1929, and following papers.

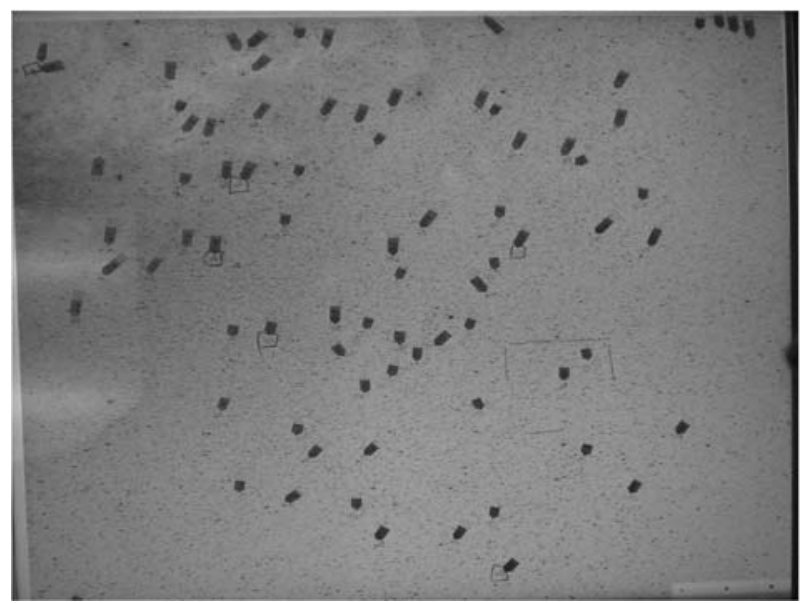

Fig. 3: One of the 290 Karl Henize southern sky Ha-alpha survey plates (Michigan-Mount Wilson Southern H-alpha survey), the bright emission objects found by Karl Henize are indicated
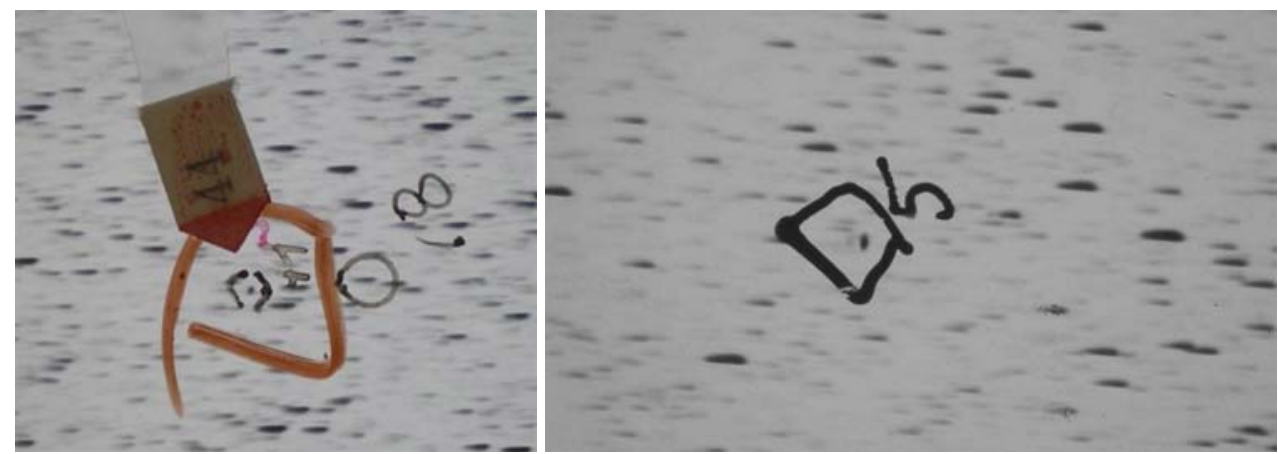

Fig. 4: Examples of objects with prominent emission lines found in the Karl Henize plate collection (Michigan-Mount Wilson Southern H-alpha survey) located at the PARI Institute, NC, USA 
(3) Hamburg Quasar Survey. A wide-angle objective prism survey searching for quasars with $B<17.5$ in the northern sky. The survey plates were taken with the former Hamburg Schmidt telescope, located at Calar Alto/Spain since 1980. Online access (http://www.hs.unihamburg.de/DE/For/Exg/Sur/index.html).

(4) Byurakan Survey. The Digitized First Byurakan Survey (DFBS) is the digitized version of the First Byurakan Survey (FBS). It is the largest spectroscopic database in the world, providing low-dispersion spectra for 20,000,000 objects on $1139 \mathrm{FBS}$ fields $=17,056 \mathrm{deg}^{2}$. Online access (http://byurakan.phys.uniroma1.it/). Sky coverage: $D E C>-15$ deg, all RA (except the Milky Way). The prism spectral plates were taken by the $1 \mathrm{~m}$ Schmidt telescope. Limiting magnitude: 17.5 in V. Spectral range: $340-690 \mathrm{~nm}$, spectral resolution $5 \mathrm{~nm}$.

(5) Spectral survey plates in the Astronomical Photographic Data Archive (APDA) located at the Pisgah Astronomical Research Institute (PARI), USA, e.g. Case QSO-Survey (http://www.pari.edu/library). Telescope: $61 / 91 \mathrm{~cm}$ Burrell Schmidt at Kitt Peak, 1.8 deg prism, plate FOV: 5-degree by 5-degree, limiting $\mathrm{B}$ magnitude: 18, emulsion: IIIaJ Baked, spectral range: $330 \mathrm{~nm}$ to $530 \mathrm{~nm}$.

(6) Karl Henize H-alpha plate collection (located since 2010 at PARI) - Michigan-Mount Wilson Southern H-alpha survey (Henize, 1954). A newly (in 2010) re-discovered highly valuable plate collection. 290 high quality plates $15 \times 15$ inches taken in 1950-1952 in South Africa by dedicated telescope by Karl Henize. Telescope aperture D25 cm, dispersion $45 \mathrm{~nm} / \mathrm{mm}$ at Halpha, various filters used (Henize, 1954).
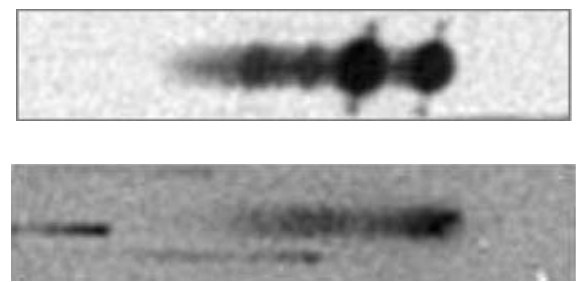

Fig. 5: Examples of objects with emission lines visible at spectral dispersion similar to Gaia BP/RP: planetary nebula (left) and UV excess galaxy (right). Source: Digitized Byurakan Survey. Note that albeit the theoretical spectral resolution of these plates is several times better than spectral resolution of Gaia $\mathrm{RP} / \mathrm{BP}$, in reality the resolution is comparable as the ground-based data are mostly affected by seeing and atmospheric influence and do not reach their theoretical values

\section{Ultra low dispersion sprectral images by Gaia $\mathbf{R P} / \mathbf{B P}$}

\subsection{Algorithms}

The algorithms for automated analyses of digitised spectral plates were developed by cumputer science students (Hudec 2007). The main goals are as follows: automated classification of spectral classes, searches for spectral variability (both continuum and lines), searches for objects with specific spectra, correlation of spectral and light changes, searches for transients, and application for Gaia. The archival spectral plates taken with the objective prism offer the possibility to simulate the Gaia low dispersion spectra and related procedures such as searches for spectral variability and variability analyses based on spectro-photometry. We focus on the sets of spectral plates of the same sky region covering long time intervals with good sampling; this enables simulation of the Gaia BP/RP outputs. The main task is automatic classification of stellar objective prism spectra on digitised plates, a simulation and a feasibility study for the low-dispersion Gaia spectra. The algorithmes developed and tested include application of novel approaches and techniques with emphasis on neural networks for automated recognition of spectral types of stars comparing them with atlas spectra and differ from techniques discussed before (e.g. Christlieb et al. 2002 or Hagen et al., 1995).

For future we plan to continue developing innovative dedicated image processing methods to continue our participation in data extraction and evaluation by providing expertise in the high level image processing with focuss on solving problems of data processing and data extraction coming from the peculiar way Gaia is functioning. The expertise available at the Department of Radioelectronics of the CTU Faculty of Electrical Engineering will be further used and developed in this direction.

\subsection{Comparison of Gaia low dispersion spectra and spectral plates}

The motivation for these studies is as follows: (1) Comparison of the simulated Gaia BP/RP images with those obtained from digitized Schmidt spectral plates (both using dispersive elements) for 8 selected test fields, and (2) Feasibility study for application for the algorithms developed for the plates for Gaia. Dispersion is an important parameter, and is discussed later: (1) Gaia BP: 4-32 nm/pixel i.e. 400-3200 nm/mm, $9 \mathrm{~nm} /$ pixel i.e. $900 \mathrm{~nm} / \mathrm{mm}$ at 
$H \gamma$, RP: $7-15 \mathrm{~nm} /$ pixel i.e. $700-1500 \mathrm{~nm} / \mathrm{mm}$. PSF FWHM $\sim 2$ px i.e. spectral resolution is $\sim 18 \mathrm{~nm}$, (2) Schmidt Sonneberg Plates (typical mean value): the dispersion for the $7 \mathrm{deg}$ prism $10 \mathrm{~nm} / \mathrm{mm}$ at $H \gamma$, and $23 \mathrm{~nm} / \mathrm{mm}$ at $H \gamma$ for the $3 \mathrm{deg}$ prism. (3) Bolivia Expedition plates: $9 \mathrm{~nm} / \mathrm{mm}$, with calibration spectrum, (4) Hamburg QSO Survey: $1.7 \mathrm{deg}$ prism, $139 \mathrm{~nm} / \mathrm{mm}$ at $H \gamma$, spectral resolution of $4.5 \mathrm{~nm}$ at $H \gamma,(5)$ Byurakan Survey: $1.5 \mathrm{deg}$ prism, $180 \mathrm{~nm} / \mathrm{mm}$ at $H \gamma$, resolution $5 \mathrm{~nm}$ at $H \gamma$ and (6) PARI prism dispersion: $150 \mathrm{~nm}$ at $450 \mathrm{~nm}$. We see that the Gaia BP/RP dispersion is $\sim 5$ to 10 times less than the dispersion of a typical digitised spectral prism plate, and the spectral resolution of Gaia is $\sim 3$ to 4 times less than for the plates (Table 1). Note that for plates the spectral resolution is seeing-limited, hence the values represent the best values, and on the plates affected by bad seeing the spectral resolution is only $\sim 2$ times better when compared to Gaia $\mathrm{BP} / \mathrm{RP}$.

\begin{tabular}{|l|l|l|l|l|}
\hline & $\begin{array}{l}\text { Wavelength } \\
\text { range, } \mathrm{mm}\end{array}$ & $\begin{array}{l}\text { Limiting } \\
\text { magnitude }\end{array}$ & $\begin{array}{l}\text { Dispersion } \\
\text { at IIgamma } \\
\mathrm{nm} / \mathrm{mm}\end{array}$ & $\begin{array}{l}\text { Spectral } \\
\text { resolution } \\
\mathrm{nm}\end{array}$ \\
\hline Gaia & $\begin{array}{l}330-660, \\
650-1000\end{array}$ & $\sim 19$ & 900 & $\sim 18$ \\
\hline $\begin{array}{l}\text { Sonneberg } \\
\text { Schmidt }\end{array}$ & $340-650$ & 18 & 10 and 23 & $\sim 3$ \\
\hline $\begin{array}{l}\text { Bolivia } \\
\text { Expedition }\end{array}$ & $340-650$ & 14 & 9 & $\sim 3$ \\
\hline $\begin{array}{l}\text { Hamburg } \\
\text { Byurakan }\end{array}$ & $340-540$ & 19 & 139 & 4.5 \\
\hline
\end{tabular}

Table 1: A comparison of Gaia RP/BP and plate lowdispersion Schmidt plate spectral surveys

\section{Examples of science with Gaia RP/BP spectro-photometry}

Despite the low dispersion discussed above, the major strength of Gaia for many scientific fields will be in spectro-photometry, as the low dispersion spectra may be transferred to numerous well-defined color filters. As an example, the Optical Afterglows (OAs) of Gamma-Ray Bursts (GRBs) are known to exhibit quite specific color indices, distiguishing them from other types of astrophysical objects (Simon et al., 2001 and 2004), hence a reliable classification of OAs of GRBs will be, in principle, possible using this method. The colors of microquasars may serve as another example: they display blue colors, with a trend of a diagonal formed by the individual objects.
This method can be used even for optically faint, and hence distant objects.

We however note that the correct color indices cannot be calculated without careful decontamination of the BP/RP spectra (Straizys et al., 2006, Straizys, 2010). The energy redistribution effect in the Gaia BP and RP spectra arising from contamination by wings of the image profiles was mentioned and investigated by Straizys et al., 2006, Montegriffo et al., 2007, and Montegriffo, 2009. According to these researchers, the Gaia spectra may be used for classifying stars either after applying contamination corrections or by using standard calibration stars with known physical parameters and observed with the Gaia spectrophotometers. In the latter case, there is no way to calculate the real spectral energy distributions, magnitudes, color indices, color excesses or other photometric quantities. The classification has to be done by matching the observed pseudo-energy distributions of the target and the standard stars, or by using pattern recognition algorithms (template matching) over the whole spectrum to estimate the astrophysical parameters of stars.
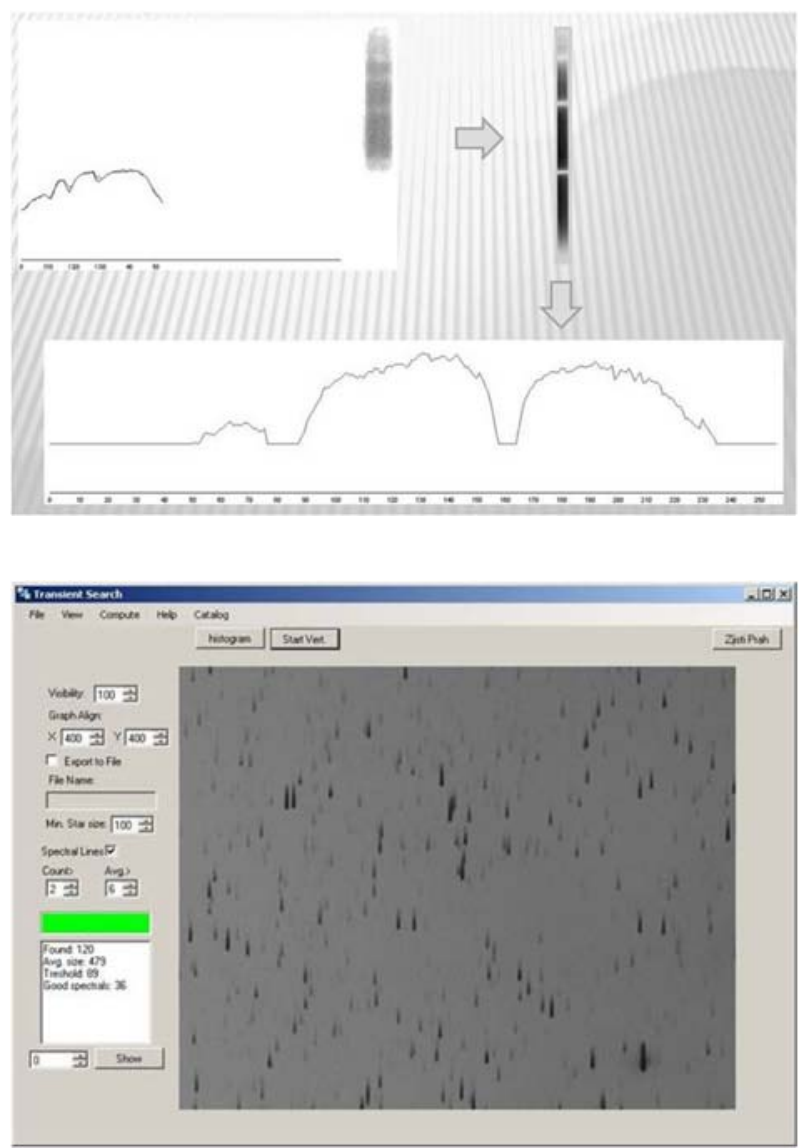

Fig. 6: Example of the automated star spectral classification process on a digitised Sonneberg Observatory lowdispersion spectral plate 


\section{Conclusion}

Variability studies based on low-dispersion spectra are expected to provide unique novel data, and can use the algorithms recently developed for automatic analyses of digitized spectral Schmidt plates. The ESA Gaia satellite will provide ultra-low dispersion spectra by $\mathrm{BP}$ and $\mathrm{RP}$, representing a new challenge for astrophysicists and for cumputer science. The nearest analogy is digitized prism spectral plates: the Sonneberg, PARI, Hamburg and Byurakan surveys. These digitised surveys can be used for a simulation and for tests of the Gaia algorithms and Gaia data. Some algorithms have already been tested. Some types of variable stars are known to exhibit large spectral type changes - however this field is little exploited and more discoveries can be expected with the Gaia data, as Gaia will allow us to investigate the spectral behavior of huge numbers of objects over 5 years with good sampling for spectroscopy. However, the data must first be decontamined to be scientifically applied, as discussed above.

\section{Acknowledgement}

Czech participation in the ESA Gaia project is supported by PECS project 98058 . The scientific part of the study is related to grants 205/08/1207 and 102/09/0997, provided by the Grant Agency of the Czech Republic. Some aspects of the project described here are a natural continuation of Czech participation in ESA INTEGRAL (ESA PECS 98023). The analyses of digitised low dispersion spectral plates are supported by MSMT KONTAKT Project ME09027.

\section{References}

[1] Becker, F.: Spektral-Durchmusterung der Kapteyn-Eichfelder des Sudhimmels. I. Pol und Zone - 75deg. Potsdam Publ., 27, 1, 1929.

[2] Henize, Karl G.: The Michigan-Mount Wilson southern Ha survey. Astronomical Journal, Vol. 59, p. 325, 1954.

[3] Hudec, L.: Algorithms for spectral classification of stars, BSc. Thesis, Charles University, Prague, 2007.
[4] Jordi, C., Carrasco, J. M.: Spectrophotometric and Polarimetric Standardization, ASP Conference Series, The Future of Photometric, 364, $215,2007$.

[5] Montegriffo, P., et al.: A model for the absolute photometric calibration of GAIA BP and RP spectra. I. Basic concepts. GAIA-C5-TNOABO-PMN-001-1, 2007.

[6] Montegriffo, P.: A model for the absolute photometric calibration of Gaia BP and RP spectra. II. Removing the LSF smearing. GAIA-C5-TNOABO-PMN-002, 2009.

[7] Šimon, V., Hudec, R., Pizzichini, G., Masetti, N.: $A \& \mathcal{E} A, \mathbf{3 7 7}, 450,2001$.

[8] Šimon, V., Hudec, R., Pizzichini, G., Masetti, N.: 30 Years of Discovery: Gamma-Ray Burst Symposium, AIP Conference Proceedings, Gamma-Ray Bursts, 727, 487-490, 2004.

[9] Šimon, V., Hudec, R., Pizzichini, G.: $A \& \mathcal{E}$, 427, 901, 2004.

[10] http://sci.esa.int/gaia/

[11] Straizys, V., et al.: Baltic Astronomy, Vol. 15, 449-459, 2006.

[12] Christlieb, N., Wisotzki, L., Grasshoff, G.: $A \&$ A, 391, 397, 2002.

[13] Hagen, H.-J., et al.: $A \& A S, \mathbf{1 1 1}, 195,1995$.

René Hudec

E-mail: rhudec@asu.cas.cz

Astronomical Institute

Academy of Sciences of the Czech Republic

CZ-251 65 Ondřejov, Czech Republic

Czech Technical University in Prague

Faculty of Electrical Engineering

Technická 2, CZ-166 27 Prague, Czech Republic

Lukáš Hudec

Miloš Klíma

Czech Technical University in Prague

Faculty of Electrical Engineering

Technická 2, CZ-166 27 Prague, Czech Republic 